Quim. Nova, Vol. 35, No. 6, 1222-1227, 2012

\title{
DETERMINAÇÃO RÁPIDA DE FÁRMACOS BÁSICOS EM PLASMA POR CROMATOGRAFIA A GÁS COM DETECTOR DE NITROGÊNIO E FÓSFORO
}

\author{
Lilian Feltraco Lizot, Luana Letícia da Silva, Bárbara Spaniol, Marina Venzon Antunes e Rafael Linden* \\ Instituto de Ciências da Saúde, Universidade Feevale, Rod. RS 239, 2755, 93352-000 Novo Hamburgo - RS, Brasil \\ Rafael Lanaro \\ Centro de Controle de Intoxicações, Faculdade de Ciências Médicas, Universidade Estadual de Campinas, Rua Tessália Vieira \\ Camargo, 126, Cidade Universitária, 13083-887 Campinas - SP, Brasil
}

Recebido em 25/8/11; aceito em 18/12/11; publicado na web em 2/3/12

\begin{abstract}
FAST DETERMINATION OF BASIC DRUGS IN PLASMA BY GAS CHROMATOGRAPHY WITH NITROGEN PHOSPHORUS DETECTOR. A simple and fast method for determination of 40 basic drugs in human plasma employing gas-chromatography with nitrogen-phosphorus detection was developed and validated. Drugs were extracted from $800 \mu \mathrm{L}$ of plasma with $250 \mu \mathrm{L}$ of butyl acetate at basic $\mathrm{pH}$. Aliquots of the organic extract were directly injected on a column with methylsilicone stationary phase. Total chromatographic run time was $25 \mathrm{~min}$. All compounds were detected in concentrations ranging from therapeutic to toxic levels, with intermediate precision CV\% below 11.2 and accuracy in the range of $92-114 \%$.
\end{abstract}

Keywords: GC-NPD; emergency toxicology; drug screening.

\section{INTRODUÇÃO}

A realização de análises toxicológicas capazes de detectar um significativo número de compostos é fundamental no contexto da toxicologia de emergência, assim como em toxicologia forense. Embora já esteja amplamente demonstrado que a identificação definitiva de uma substância requer a utilização associada de múltiplos métodos analíticos, ${ }^{1}$ a cromatografia a gás (CG) empregando colunas capilares possui grande aplicabilidade na identificação e quantificação de fármacos em amostras biológicas, com diversos métodos já descritos. ${ }^{2-8} \mathrm{~A}$ elevada reprodutibilidade dos parâmetros de retenção, quando adequadamente expressos, permite a identificação de um grande número de substâncias com base em bancos de dados interlaboratoriais, especialmente no contexto da análise toxicológica sistemática (ATS), devido à disponibilidade de um banco de dados com índices de retenção de aproximadamente 4.000 substâncias. ${ }^{9}$ Entretanto, não existem publicações que descrevam a associação da abordagem qualitativa empregando este banco de dados com uma estratégia de quantificação de múltiplos analitos, especialmente considerando que a determinação quantitativa é fundamental visto que a maioria das substâncias possui efeitos tóxicos dependentes de suas concentrações plasmáticas. ${ }^{10}$

Recentemente, diversos métodos para identificação e quantificação de múltiplos analitos em sangue, plasma ou soro em toxicologia clínica e forense foram descritos, baseados principalmente em cromatografia líquida associada à espectrometria de massas. ${ }^{11-14}$ A intenção destes métodos multianalitos é monitorar múltiplas substâncias de interesse com uma única preparação de amostra e procedimento de análise. Os métodos multianalitos em toxicologia analíticos são preferíveis porque tornam o processo analítico mais simples, rápido, barato e permitem o monitoramento de analitos de diferentes classes terapêuticas em uma única amostra corporal. Isto é relevante porque frequentemente somente uma quantidade limitada de amostra está disponível e o número total de analitos na amostra é desconhecido. Uma alternativa útil e de menor custo para a determinação de múltiplos analitos em um único método é o emprego da

*e-mail: rafael.linden@feevale.br
CG-DNP. Dentre as características vantajosas da CG para análises toxicológicas multianalitos estão o baixo custo operacional e a eficiência de separação. A estas qualidades soma-se a sensibilidade e especificidade do detector seletivo de nitrogênio e fósforo (DNP). De fato, considerando que aproximadamente $90 \%$ das substâncias de interesse toxicológico podem ser detectadas pelo DNP e a sua relativa insensibilidade a compostos endógenos usuais, a associação CG-DNP é de especial utilidade em análises toxicológicas em bioamostras. ${ }^{6}$ Estas características permitem a injeção direta de extratos orgânicos de amostras biológicas, sem uma etapa de evaporação de solvente, mantendo a sensibilidade necessária para a identificação de substâncias em concentrações toxicologicamente relevantes. Desta forma, a associação proposta permite cumprir a orientação da Academia Nacional de Bioquímica Clínica dos Estados Unidos da América do Norte, que recomenda que testes de triagem toxicológica devem ter seus resultados disponibilizados aos serviços de emergência médica em até 4 h após a coleta das amostras biológicas. ${ }^{15}$

Desta forma, o objetivo do presente estudo foi desenvolver e validar um método para a determinação de 40 fármacos de caráter básico e relevância toxicológica, associando uma preparação de amostra simples e rápida a um sistema de separação em cromatografia a gás com elevada comparabilidade interlaboratorial, apresentando características adequadas para utilização em toxicologia de emergência. $\mathrm{O}$ trabalho descreve de forma pioneira a introdução de um procedimento quantitativo a um método de identificação de substâncias baseado em índices de retenção em CG previamente descrito.

\section{PARTE EXPERIMENTAL}

\section{Materiais e reagentes}

Os padrões de fármacos foram obtidos de diversas indústrias farmacêuticas ou da Cerilliant (Round Rock, EUA) e suas estruturas químicas estão disponíveis na Figura 1S, material suplementar. Metanol grau cromatográfico e acetato de butila foram provenientes da Mallinckrodt Baker (Phillipsburg, EUA). Tris-(hidroximetil)aminometano e hidróxido de sódio foram provenientes da Nuclear (Diadema, Brasil). Água ultrapurificada foi obtida através de um 
sistema Elga Purelab Ultra, proveniente da Elga Labwater (High Wycombe, Reino Unido).

\section{Soluções}

Foram preparadas soluções estoque (SE) de todos os analitos através da dissolução de $10 \mathrm{mg}$ da substância, na forma de base, em $10 \mathrm{~mL}$ de metanol. Através da mistura de diferentes SE e da diluição com metanol, foram obtidas 5 soluções de trabalho (ST1), com concentração final dos analitos de $100 \mu \mathrm{g} \mathrm{mL} \mathrm{mL}^{-1}$. A mistura A (ST1 A) conteve bromazepam, buspirona, ciclobenzaprina, diazepam, efedrina, maprotilina, sibutramina, tioridazina e tranilcipromina. A mistura B (ST1 B) conteve amitriptilina, benzocaína, dexfenfluramina, estricnina, haloperidol, nordazepam, nitrazepam, sertralina e venlafaxina. A mistura C (ST1 C) conteve anfepramona, codeína, clomipramina, fenfluramina, fluoxetina, flurazepam, imipramina, levomepromazina e tri-hexafenidila. A mistura D (ST1 D) conteve citalopram, dextrometorfano, femproporex, mazindol, paroxetina e zolpidem. A mistura E (ST1 E) conteve cafeína, clometiazol, lidocaína, carbamazepina, cinarizina, nortriptilina e tramadol. Todas as ST1 (A, B, C, D e E) foram diluídas com metanol a fim de se obter soluções nas concentrações $40 \mu \mathrm{g} \mathrm{mL}^{-1}$ (ST2), $20 \mu \mathrm{g} \mathrm{mL}^{-1}$ (ST3), 10 $\mu \mathrm{g} \mathrm{mL}^{-1}$ (ST4) e $4 \mu \mathrm{g} \mathrm{mL}^{-1}$ (ST5). A solução de trabalho do padrão interno (PI, proadifen, $20 \mu \mathrm{g} \mathrm{mL}^{-1}$ ) foi preparada através da diluição da solução estoque com metanol. A solução tampão $\mathrm{pH} 10$ foi preparada através da dissolução de 23,4 g de tris-(hidroximetil)-aminometano em $1000 \mathrm{~mL}$ de água ultrapurificada, com posterior ajuste do $\mathrm{pH}$ com solução de $\mathrm{NaOH} 0,1 \mathrm{~mol} \mathrm{~L}^{-1}$.

\section{Equipamento e condições cromatográficas}

As análises foram realizadas em um cromatógrafo a gás CP 3800, equipado com um amostrador automático multiuso Varian ${ }^{\circledR}$ PAL e detector de nitrogênio e fósforo, controlados pelo programa Galaxie ${ }^{\circledR}$, todos provenientes da Varian ${ }^{\circledR}$ (Middelburg, Holanda). A separação foi realizada em uma coluna OV-1 (30 m x 0,32 mm, $1 \mu \mathrm{m}$ de espessura de filme) contendo metilsilicone como fase estacionária, proveniente da Ohio Valley (Marrietta, EUA). O tempo total de análise cromatográfica foi de $25 \mathrm{~min}$. A temperatura inicial da coluna foi de $140{ }^{\circ} \mathrm{C}$, mantida por $2 \mathrm{~min}$, seguida de um incremento de temperatura de 10 ${ }^{\circ} \mathrm{C}$ por min até atingir $320{ }^{\circ} \mathrm{C}$, sendo esta temperatura mantida por 5 min. O gás de arraste utilizado foi o hélio, proveniente da Linde Gases (Esteio, Brasil) sob fluxo constante de $4 \mathrm{~mL} \mathrm{~min}^{-1}$. O volume de injeção foi de $2 \mu \mathrm{L}$, com injetor mantido a $260{ }^{\circ} \mathrm{C}$ no modo de injeção sem divisão de fluxo. $\mathrm{O}$ detector foi mantido a $310^{\circ} \mathrm{C}$, com corrente de $3,2 \mathrm{pA}$.

\section{Reprodutibilidade dos parâmetros de retenção}

Os parâmetros de retenção cromatográfica avaliados foram: tempo de retenção absoluto $\left(t_{R}\right)$, tempo de retenção relativo $\left(t_{R R}\right)$ e índice de retenção (IR). Estes parâmetros foram determinados em 20 dias não consecutivos e, a partir dos dados obtidos, foram calculados a média, o desvio padrão e coeficiente de variação percentual (CV\%) para cada parâmetro da série. Os $t_{\mathrm{RR}}$ foram calculados utilizando a Equação 1 , onde $t_{x}$ é o tempo de retenção da substância desconhecida e $t_{p i}$ é o tempo de retenção do padrão interno. Os IR foram determinados através da Equação 2, na qual os tempos de retenção e os índices de retenção das substâncias de referência eluindo imediatamente antes e depois da substância desconhecida são denotados $t_{n}, t_{n+1}, I_{n}$ e IR $R_{n+1}$, respectivamente, e t é o tempo de retenção da substância cujo IR será calculado. Para determinação do IR foi empregada como referência uma mistura contendo 10 substâncias em metanol na concentração de $100 \mu \mathrm{g} \mathrm{mL}^{-1}$ (solução de calibração de índices de retenção). Esta solução conteve fenfluramina ( $I R=1230)$, efedrina ( $I R=1365)$, cafeína (IR = 1800), venlafaxina (IR = 2083), amitriptilina (IR = 2194), ciclobenzaprina ( $I R=2233$ ), citalopram ( $I R=2380$ ), levomepromazina ( $I R=2525)$, flurazepam ( $I R=2780$ ) e buspirona (IR =3221). Em cada dia do experimento foi injetado $1 \mu \mathrm{L}$ da solução de trabalho de proadifen, da solução de calibração de índices de retenção e das cinco diferentes soluções de trabalho.

$$
\begin{gathered}
t_{R R x}=\frac{t_{x}}{t_{p i}} \\
I R_{x}=\left(I R_{n+1}-I R_{n}\right) \frac{\left(t_{x}-t_{n}\right)}{\left(t_{n+1}-t_{n}\right)}+I R_{n}
\end{gathered}
$$

\section{Preparação das amostras}

As amostras foram preparadas em microtubos de polipropileno de 1,5 mL, adicionando-se $800 \mu \mathrm{L}$ de plasma (amostra clínica, solução analítica ou controle), $250 \mu \mathrm{L}$ de solução $\mathrm{pH} 10,50 \mu \mathrm{L}$ de solução de trabalho de PI (proadifen, $20 \mu \mathrm{g} \mathrm{mL}^{-1}$ ) e $250 \mu \mathrm{L}$ de acetato de butila. A mistura foi homogeneizada em vórtex por $40 \mathrm{~s}$ e, em seguida, centrifugada a $12.000 \mathrm{~g}$ por $20 \mathrm{~min}$. A fase orgânica foi transferida para um frasco do amostrador automático, o qual foi inserido no equipamento para posterior análise cromatográfica.

\section{Especificidade}

A especificidade foi avaliada através do processamento, com o método descrito, de 6 amostras de plasma de voluntários que não estavam utilizando quaisquer medicamentos, a fim de se avaliar a presença de picos endógenos.

\section{Linearidade}

A partir da diluição de $40 \mu \mathrm{L}$ das ST1, ST2, ST3, ST4 e ST5 com $760 \mu \mathrm{L}$ de plasma foram obtidas soluções analíticas nas concentrações $5,2,1,0,5$ e $0,2 \mu \mathrm{g} \mathrm{mL} \mathrm{m}^{-1}$ dos diferentes fármacos, com exceção dos fármacos cafeína, carbamazepina e lidocaína, cujas soluções analíticas foram preparadas na faixa de 2 a $50 \mu \mathrm{g} \mathrm{mL}^{-1}$. As soluções analíticas foram analisadas em quadruplicata e as curvas analíticas foram construídas calculando-se a razão entre as áreas dos picos dos diferentes analitos e a área do pico do padrão interno (y) e comparando-se estas razões com as concentrações nominais das soluções analíticas (x). As curvas foram ajustadas através do método dos mínimos quadrados empregando regressão linear ponderada, com fator ponderal 1/x.

\section{Precisão e exatidão}

As amostras de controle de qualidade foram preparadas através de adição de $80 \mu \mathrm{L}$ das ST2 e ST5 a $720 \mu \mathrm{L}$ de plasma a fim de

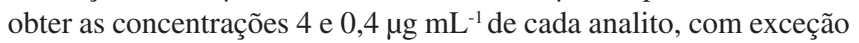
dos fármacos cafeína, carbamazepina e lidocaína, para os quais as concentração das amostras controle foram 40 e $4 \mu \mathrm{g} \mathrm{mL}^{-1}$ (controle de qualidade baixo, CQB e controle de qualidade alto, CQA). As amostras de controle de qualidade foram analisadas como descrito no item Preparação de amostras, em triplicata em 3 dias diferentes. As precisões intra- e interensaios foram determinadas por ANOVA com a variável agrupadora "dia" e foram expressas como CV\%. A exatidão foi definida como a percentagem da concentração nominal representada pela concentração calculada pela curva analítica. O critério de aceitação para a exatidão foram valores dentro de uma faixa de $\pm 15 \%$ do valor nominal e para precisão foi de um CV de até $15 \%{ }^{16}$ 


\section{Aplicação do método}

O método foi aplicado a 8 amostras de pacientes atendidos no serviço de emergência médica de um hospital geral, suspeitos de intoxicações. As amostras de sangue foram obtidas por punção venosa para tubos com EDTA, o plasma foi separado por centrifugação e o plasma foi mantido a $-20{ }^{\circ} \mathrm{C}$ até a análise.

\section{RESULTADOS E DISCUSSÃO}

A aplicação da CG para análise toxicológica sistemática e identificação de fármacos em toxicologia clínica e forense requer a utilização de parâmetros de retenção de elevada precisão, sendo que diferentes formas de expressar a retenção têm sido utilizadas. A separação cromatográfica empregou fase estacionária metilsilicone, que permitiu uma adequada resolução e dispersão de retenção dos diferentes analitos em um tempo razoável. Conforme apresentado na Tabela 1, o mais impreciso dos parâmetros de retenção avaliados foi $\mathrm{ot}_{\mathrm{R}}$, com CV\% médio de 0,34 (faixa de 0,11 a 1,83 ). Uma alternativa para aumentar a precisão é expressar a retenção relacionada a uma substância de referência, o $\mathrm{t}_{\mathrm{RR}}$. Empregou-se proadifen como padrão interno, tanto para retenção como para quantificação dos analitos, visto apresentar tempo de retenção intermediário (média de 15,34 min), não ser utilizado como fármaco ou droga de abuso e apresentar comportamento cromatográfico adequado. $\mathrm{Ot}_{\mathrm{RR}}$ apresentou um $\mathrm{CV} \%$ médio de 0,301 (faixa de 0,061 a 1,084), levemente inferior ao $t_{R}$.

Tabela 1. Reprodutibilidade dos parâmetros de retenção de 40 fármacos básicos $(\mathrm{n}=20)$

\begin{tabular}{|c|c|c|c|c|c|c|c|c|c|c|}
\hline \multirow{2}{*}{ Substância } & \multicolumn{3}{|c|}{ Tempo de retenção (min) } & \multicolumn{3}{|c|}{ Tempo de retenção relativo } & \multicolumn{4}{|c|}{ Índice de retenção } \\
\hline & Média & $\mathrm{DP}$ & $\mathrm{CV} \%$ & Média & DP & $\mathrm{CV} \%$ & Média & Referência $^{7}$ & $\mathrm{DP}$ & $\mathrm{CV} \%$ \\
\hline Amitriptilina* & 13,83 & 0,02 & 0,11 & 0,932 & 0,001 & 0,093 & 2194 & 2194 & - & - \\
\hline Anfepramona & 6,63 & 0,03 & 0,46 & 0,447 & 0,002 & 0,403 & 1502 & n.d. & 1,5 & 0,10 \\
\hline Benzocaína & 7,15 & 0,03 & 0,41 & 0,482 & 0,002 & 0,371 & 1549 & 1545 & 2,5 & 0,16 \\
\hline Bromazepam & 17,09 & 0,14 & 0,79 & 1,152 & 0,009 & 0,787 & 2660 & 2665 & 0,9 & 0,04 \\
\hline Buspirona* & 22,23 & 0,04 & 0,19 & 1,498 & 0,002 & 0,160 & 3221 & 3221 & - & - \\
\hline Cafeína* & 9,95 & 0,04 & 0,35 & 0,670 & 0,002 & 0,285 & 1800 & 1800 & - & - \\
\hline Carbamazepina & 14,61 & 0,02 & 0,13 & 0,984 & 0,001 & 0,085 & 2283 & 2285 & 1,6 & 0,07 \\
\hline Ciclobenzaprina* & 14,18 & 0,03 & 0,19 & 0,956 & 0,001 & 0,141 & 2195 & 2195 & - & - \\
\hline Cinarizina & 20,38 & 0,02 & 0,11 & 1,374 & 0,001 & 0,086 & 3011 & n.d. & 1,5 & 0,03 \\
\hline Citalopram* & 15,46 & 0,02 & 0,12 & 1,042 & 0,001 & 0,090 & 2380 & 2380 & - & 0,05 \\
\hline Clometiazol & 3,79 & 0,02 & 0,63 & 0,256 & 0,002 & 0,679 & 1234 & 1227 & 0,1 & 0,07 \\
\hline Clomipramina & 15,67 & 0,03 & 0,18 & 1,056 & 0,001 & 0,134 & 2407 & 2415 & 1,8 & 0,01 \\
\hline Codeina & 15,42 & 0,03 & 0,17 & 1,039 & 0,001 & 0,096 & 2374 & 2375 & 1,4 & 0,07 \\
\hline Dexfenfluramina & 3,81 & 0,05 & 1,27 & 0,256 & 0,004 & 1,382 & 1236 & n.d. & 1,2 & 0,06 \\
\hline Dextrometorfano & 13,35 & 0,02 & 0,15 & 0,900 & 0,001 & 0,064 & 2141 & 2145 & 1,8 & 0,10 \\
\hline Diazepam & 15,81 & 0,03 & 0,16 & 1,065 & 0,002 & 0,162 & 2425 & 2428 & 2,4 & 0,09 \\
\hline Efedrina* & 5,12 & 0,03 & 0,51 & 0,345 & 0,002 & 0,525 & 1365 & 1365 & - & - \\
\hline Estricnina & 21,22 & 0,06 & 0,28 & 1,430 & 0,003 & 0,221 & 3109 & 3116 & 5,5 & 0,10 \\
\hline Femproporex & 7,53 & 0,02 & 0,23 & 0,508 & 0,001 & 0,122 & 1583 & n.d. & 1,2 & 0,18 \\
\hline Fenfluramina* & 3,76 & 0,05 & 1,41 & 0,254 & 0,004 & 1,427 & 1230 & 1230 & - & - \\
\hline Fluoxetina & 10,51 & 0,02 & 0,22 & 0,707 & 0,001 & 0,135 & 1856 & 1859 & 1,7 & 0,11 \\
\hline Flurazepam* & 18,37 & 0,03 & 0,13 & 1,238 & 0,001 & 0,094 & 2780 & 2780 & - & 0,09 \\
\hline Haloperidol & 19,59 & 0,03 & 0,13 & 1,320 & 0,001 & 0,075 & 2925 & 2930 & 1,2 & 0,04 \\
\hline Imipramina & 14,06 & 0,03 & 0,23 & 0,948 & 0,002 & 0,172 & 2220 & 2230 & 0,1 & 0,04 \\
\hline Levomepromazina* & 16,56 & 0,02 & 0,15 & 1,116 & 0,001 & 0,124 & 2525 & 2525 & - & - \\
\hline Lidocaína & 10,73 & 0,02 & 0,14 & 0,723 & 0,000 & 0,063 & 1880 & 1870 & 1,4 & 0,06 \\
\hline Maprotilina & 15,05 & 0,02 & 0,14 & 1,014 & 0,001 & 0,107 & 2352 & 2356 & 1,4 & 0,08 \\
\hline Mazindol & 15,07 & 0,02 & 0,14 & 1,016 & 0,001 & 0,097 & 2346 & 2325 & 2,1 & 0,06 \\
\hline Nitrazepam & 18,11 & 0,02 & 0,13 & 1,222 & 0,001 & 0,061 & 2745 & 2740 & 1,3 & 0,09 \\
\hline Nordazepam & 16,25 & 0,02 & 0,13 & 1,095 & 0,002 & 0,153 & 2484 & 2490 & 2,1 & 0,05 \\
\hline Nortriptilina & 13,99 & 0,02 & 0,15 & 0,942 & 0,001 & 0,106 & 2213 & 2215 & 0,7 & 0,09 \\
\hline Paroxetina & 16,81 & 0,31 & 1,83 & 1,133 & 0,021 & 1,824 & 2570 & 2580 & 1,6 & 0,06 \\
\hline Sertralina & 15,32 & 0,02 & 0,12 & 1,032 & 0,001 & 0,092 & 2369 & 2368 & 1,1 & 0,05 \\
\hline Sibutramina & 10,63 & 0,02 & 0,16 & 0,717 & 0,000 & 0,055 & 1869 & n.d. & 1,5 & 0,08 \\
\hline Tioridazina & 20,95 & 0,03 & 0,14 & 1,412 & 0,002 & 0,114 & 3096 & 3115 & 1,8 & 0,06 \\
\hline Tramadol & 11,44 & 0,02 & 0,19 & 0,771 & 0,001 & 0,083 & 1951 & 1943 & 1,5 & 0,08 \\
\hline Tranilcipromina & 3,61 & 0,04 & 1,05 & 0,243 & 0,003 & 1,084 & 1221 & 1220 & 1,0 & 0,09 \\
\hline Trihexafenidila & 14,37 & 0,03 & 0,18 & 0,968 & 0,001 & 0,125 & 2252 & 2245 & 1,7 & 0,08 \\
\hline Venlafaxina* & 12,73 & 0,02 & 0,13 & 0,858 & 0,001 & 0,092 & 2083 & 2083 & - & - \\
\hline Zolpidem & 18,36 & 0,02 & 0,11 & 1,237 & 0,001 & 0,086 & 2780 & 2780 & 1,5 & 0,05 \\
\hline Média geral & & & 0,34 & & & 0,301 & & & & 0,07 \\
\hline
\end{tabular}

*Substâncias presentes na mistura de calibração de IR (desvio padrão e CV\% não calculados). n.d.: não disponível na ref. 7. 
A utilização de uma mistura de fármacos para determinar IR (Figura 1) como forma de expressar a retenção permitiu obter uma precisão marcadamente superior, com CV\% médio de 0,07 (faixa de 0,01 a 0,18). Além disto, os valores de IR obtidos foram semelhantes àqueles disponíveis em um grande banco de dados de retenção de substâncias de interesse toxicológico publicado pela The International Association of Forensic Toxicologists (TIAFT) em 1992, demonstrando também sua utilidade interlaboratorial. ${ }^{9}$ As diferenças entre os valores de IR determinados e os existentes na publicação da TIAFT foram de 0 a 21 unidades de IR, dentro da janela de busca recomendada de \pm 25 unidades para identificação de substâncias por CG em ATS. ${ }^{1}$ Desta forma, o método proposto pode também indicar a presença de substâncias que não foram testadas na validação do método, mas que estão presentes no banco de dados da TIAFT, especialmente quando associados a outros métodos de análise e um processamento computacional adequado. ${ }^{1,17}$ Esta característica é especialmente útil quando dados clínicos do paciente estão disponíveis, o que pode dispensar a necessidade de uma identificação quimicamente inequívoca. Também se destaca que a determinação dos índices de retenção empregou uma mistura de fármacos amplamente disponíveis como referência, diferentemente de outros autores que utilizaram séries homólogas não disponíveis comercialmente. ${ }^{4,6,7}$
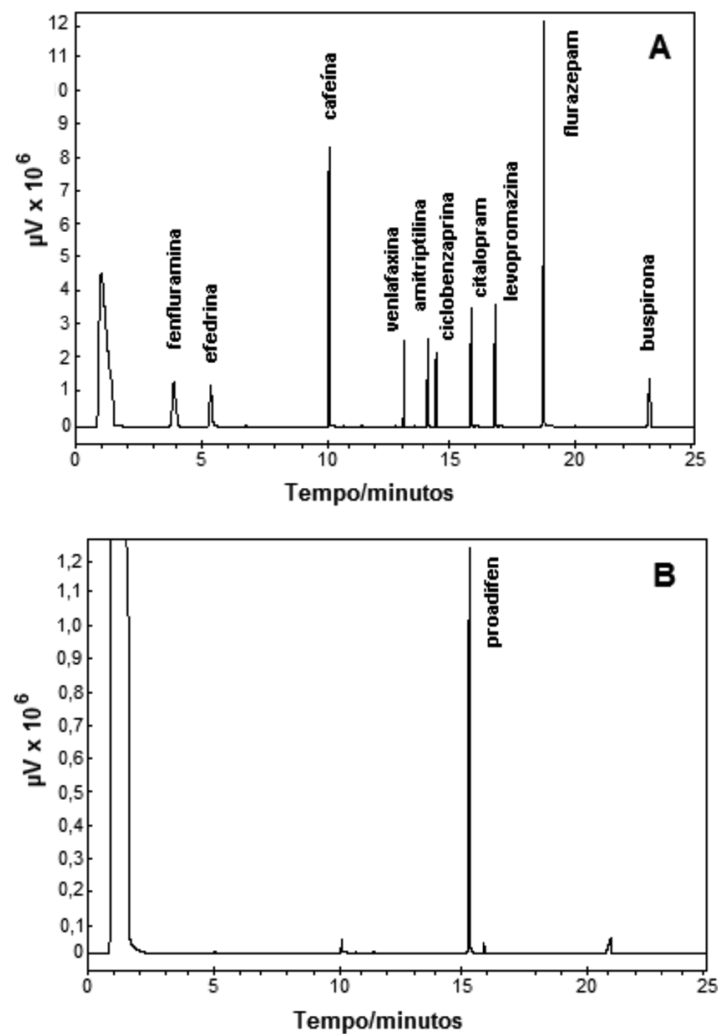

Figura 1. A: solução de calibração de índices de retenção. B: amostra de plasma "branco" adicionada de padrão interno, conforme descrito na preparação das amostras

A preparação das amostras utilizou um procedimento simples e rápido de extração líquido-líquido, sem etapa de evaporação de solvente, o qual se mostrou efetivo para os fármacos avaliados. Análises de amostras isentas de fármacos apresentaram poucos picos interferentes, com áreas relativamente pequenas, os quais tiveram tempos de retenção médios de 5,03; 10,18; 15,81 e 21,32 min (Figura 1). Apenas o pico endógeno de 15,81 min apresenta coeluição com um dos fármacos avaliados, o diazepam, sendo que as áreas determinadas apresentam contribuição insignificante na quantificação deste composto nas concentrações usualmente encontradas. A sensibilidade e a especificidade com respeito aos interferentes endógenos caracterizam a utilidade do detector de nitrogênio e fósforo na determinação de fármacos em bioamostras. Além disto, a estratégia de preparação de amostra empregada, associada a um tempo de análise cromatográfica de 25 min, permitiu que todo o ciclo analítico de uma amostra fosse realizado em aproximadamente $35 \mathrm{~min}$. Este tempo rápido de análise é fundamental especialmente no contexto da toxicologia de emergência, onde os resultados podem ser utilizados para determinar condutas terapêuticas.

A Tabela 2 apresenta os resultados dos estudos de validação para os 40 fármacos avaliados neste estudo. As faixas lineares foram ajustadas de acordo com as concentrações esperadas destas substâncias, tanto em utilizações terapêuticas como tóxicas, com coeficientes de determinação entre 0,9811 a 0,999 . A precisão intraensaios apresentou valores de CV\% entre 3,4 e 12,5 para o CQB e de 1,9 a 9,5 para o CQA. A precisão intermediária apresentou CV\% entre 3,3 e 11,2\% para o CQB e de 2,8 a 10,3 para o CQA, todos dentro dos limites de aceitação para métodos bioanalíticos. ${ }^{16} \mathrm{~A}$ exatidão também apresentou valores aceitáveis, entre 96 e 109\% para o CQB e de 92 a 109\% para o CQA, especialmente considerando que o propósito deste desenvolvimento é o diagnóstico de intoxicações, onde as concentrações encontradas tendem a ser marcadamente superiores às mensuradas no uso terapêutico de fármacos.

O método foi aplicado a 8 amostras provenientes de pacientes com suspeita de intoxicação por fármacos, atendidos em um serviço de urgência médica de um hospital universitário (Tabela 3). Nestes casos foram identificadas e quantificadas as substâncias amitriptilina (1 amostra), femproporex (1 amostra) e carbamazepina (6 amostras), sendo que 3 pacientes apresentaram concentrações de carbamazepina superiores a $10 \mu \mathrm{g} / \mathrm{mL}$, o que pode caracterizar uma exposição tóxica (Figura 2). Cabe destacar que os valores calculados de $\mathrm{T}_{\mathrm{RR}} \mathrm{e}$
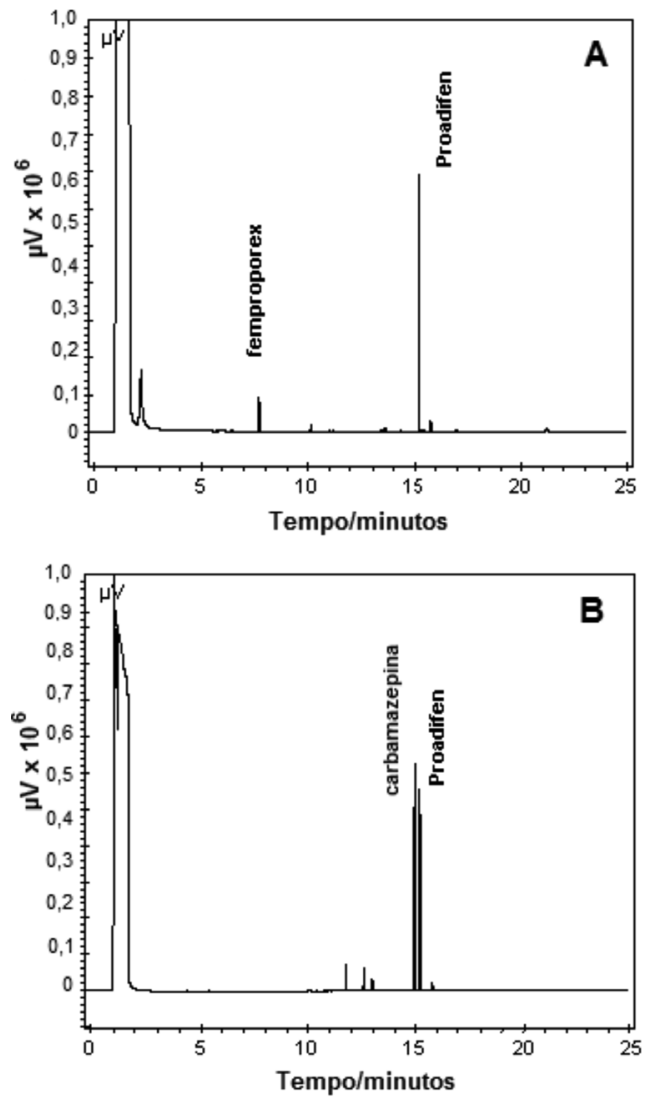

Figura 2. A: amostra de paciente contendo femproporex $\left(0,21 \mu \mathrm{g} m L^{-1}\right)$. B: amostra de paciente contendo carbamazepina $\left(20,51 \mu \mathrm{g} \mathrm{mL}^{-1}\right)$ 
Tabela 2. Dados de validação para 40 fármacos básicos

\begin{tabular}{|c|c|c|c|c|c|c|c|c|c|}
\hline \multirow[t]{2}{*}{ Substância } & \multirow{2}{*}{$\begin{array}{l}\text { Intervalo linear } \\
\qquad\left(\mu \mathrm{g} \mathrm{mL} L^{-1}\right)\end{array}$} & \multirow[t]{2}{*}{ Curva analítica } & \multirow[t]{2}{*}{$r^{2}$} & \multicolumn{2}{|c|}{$\begin{array}{c}\text { Precisão intraensaios } \\
(\mathrm{CV} \%)\end{array}$} & \multicolumn{2}{|c|}{$\begin{array}{l}\text { Precisão intermediária } \\
(\mathrm{CV} \%)\end{array}$} & \multicolumn{2}{|c|}{ Exatidão (\%) } \\
\hline & & & & CQB* & $\mathrm{CQA}^{* *}$ & $\mathrm{CQB}^{*}$ & CQA** & CQB* & CQA** \\
\hline Amitriptilina & $0,2-5,0$ & $y=0,884 x-0,085$ & 0,9990 & 4,4 & 2,1 & 3,3 & 4,1 & 109 & 103 \\
\hline Anfepramona & $0,2-5,0$ & $y=0,748 x-0,076$ & 0,9930 & 5,5 & 2,5 & 5,7 & 3,6 & 98 & 102 \\
\hline Benzocaína & $0,2-5,0$ & $\mathrm{y}=0,312 \mathrm{x}-0,079$ & 0,9877 & 7,8 & 3,1 & 7,7 & 4,5 & 93 & 98 \\
\hline Bromazepam & $0,2-5,0$ & $y=0,355 x-0,066$ & 0,9911 & 7,1 & 5,9 & 6,6 & 5,3 & 92 & 97 \\
\hline Buspirona & $0,2-5,0$ & $y=1,140 x-0,012$ & 0,9995 & 10,1 & 6,3 & 9,5 & 7,5 & 108 & 103 \\
\hline Cafeína & $2,0-50,0$ & $y=1,080 x-0,161$ & 0,9980 & 12,5 & 7,4 & 11,1 & 8,2 & 93 & 102 \\
\hline Carbamazepina & $2,0-50,0$ & $y=0,057 x-0,022$ & 0,9990 & 6,9 & 3,1 & 5,8 & 5,1 & 95 & 99 \\
\hline Ciclobenzaprina & $0,2-5,0$ & $y=0,463 x+0,031$ & 0,9998 & 6,1 & 3,9 & 7,3 & 5,8 & 96 & 103 \\
\hline Cinarizina & $0,2-5,0$ & $y=0,680 x-0,144$ & 0,9850 & 12,2 & 3,0 & 11,2 & 4,1 & 92 & 101 \\
\hline Citalopram & $0,2-5,0$ & $y=0,775 x-0,026$ & 0,9990 & 3,4 & 3,9 & 4,1 & 4,3 & 97 & 100 \\
\hline Clometiazol & $0,2-5,0$ & $y=0,746 x-0,087$ & 0,9910 & 9,1 & 3,8 & 8,8 & 4,1 & 95 & 106 \\
\hline Clomipramina & $0,2-5,0$ & $y=1,057 x-0,026$ & 0,9990 & 4,5 & 1,9 & 5,1 & 2,8 & 97 & 101 \\
\hline Codeina & $0,2-5,0$ & $\mathrm{y}=1,011 \mathrm{x}+0,025$ & 0,9960 & 4,6 & 7,1 & 5,3 & 5,2 & 104 & 106 \\
\hline Dexfenfluramina & $0,2-5,0$ & $y=0,114 x+0,034$ & 0,9981 & 7,8 & 6,1 & 5,8 & 7,2 & 106 & 98 \\
\hline Dextrometorfano & $0,2-5,0$ & $y=0,389 x+0,010$ & 0,9970 & 4,6 & 6,9 & 5,1 & 7,4 & 105 & 103 \\
\hline Diazepam & $0,2-5,0$ & $y=1,308 x+0,091$ & 0,9999 & 7,3 & 8,8 & 6,9 & 9,2 & 110 & 98 \\
\hline Efedrina & $0,2-5,0$ & $y=0,312 x+0,055$ & 0,9853 & 11,3 & 9,5 & 10,8 & 7,7 & 114 & 107 \\
\hline Estricnina & $0,2-5,0$ & $\mathrm{y}=0,051 \mathrm{x}+0,033$ & 0,9811 & 10,8 & 6,1 & 9,5 & 6,5 & 112 & 107 \\
\hline Femproporex & $0,2-5,0$ & $y=0,503 x+0,037$ & 0,9960 & 3,8 & 4,4 & 3,5 & 4,9 & 95 & 99 \\
\hline Fenfluramina & $0,2-5,0$ & $y=0,315 x+0,086$ & 0,9861 & 7,1 & 2,5 & 7,3 & 3,1 & 104 & 102 \\
\hline Fluoxetina & $0,2-5,0$ & $y=0,307 x+0,024$ & 0,9930 & 9,2 & 7,9 & 8,5 & 8,8 & 107 & 103 \\
\hline Flurazepam & $0,2-5,0$ & $\mathrm{y}=1,117 \mathrm{x}-0,071$ & 0,9920 & 10,3 & 1,9 & 8,5 & 3,1 & 96 & 98 \\
\hline Haloperidol & $0,2-5,0$ & $y=0,163 x+0,088$ & 0,9901 & 8,9 & 3,3 & 9,1 & 5,4 & 108 & 103 \\
\hline Imipramina & $0,2-5,0$ & $y=1,066 x-0,044$ & 0,9990 & 9,3 & 2,3 & 8,5 & 3,4 & 99 & 101 \\
\hline Levomepromazina & $0,2-5,0$ & $y=0,788 x-0,055$ & 0,9891 & 9,7 & 2,7 & 8,7 & 5,3 & 97 & 103 \\
\hline Lidocaína & $2,0-50,0$ & $y=0,883 x-0,053$ & 0,9990 & 8,2 & 4,8 & 7,9 & 4,6 & 97 & 99 \\
\hline Maprotilina & $0,2-5,0$ & $y=0,673 x-0,033$ & 0,9885 & 9,0 & 8,8 & 8,3 & 7,7 & 104 & 105 \\
\hline Mazindol & $0,2-5,0$ & $y=0,291 x-0,026$ & 0,9970 & 6,9 & 4,3 & 5,7 & 4,8 & 97 & 104 \\
\hline Nitrazepam & $0,2-5,0$ & $y=0,436 x+0,074$ & 0,9970 & 7,4 & 8,5 & 7,8 & 7,9 & 109 & 98 \\
\hline Nordazepam & $0,2-5,0$ & $y=1,010 x-0,098$ & 0,9997 & 6,8 & 4,7 & 6,5 & 5,3 & 94 & 105 \\
\hline Nortriptilina & $0,2-5,0$ & $y=0,798 x-0,093$ & 0,9934 & 5,9 & 6,3 & 6,6 & 5,1 & 102 & 106 \\
\hline Paroxetina & $0,2-5,0$ & $y=0,218 x-0,045$ & 0,9821 & 7,8 & 5,2 & 7,2 & 4,9 & 107 & 97 \\
\hline Sertralina & $0,2-5,0$ & $y=0,312 x-0,033$ & 0,9980 & 8,3 & 3,5 & 7,1 & 4,2 & 96 & 102 \\
\hline Sibutramina & $0,2-5,0$ & $y=0,661 x+0,011$ & 0,9993 & 8,8 & 8,2 & 7,6 & 8,3 & 98 & 104 \\
\hline Tioridazina & $0,2-5,0$ & $y=0,571 x+0,024$ & 0,9990 & 6,5 & 7,2 & 8,1 & 7,3 & 108 & 102 \\
\hline Tramadol & $0,2-5,0$ & $y=0,102 x+0,069$ & 0,9980 & 6,7 & 2,2 & 6,6 & 5,3 & 95 & 105 \\
\hline Tranilcipromina & $0,2-5,0$ & $y=0,427 x+0,073$ & 0,9835 & 11,8 & 7,1 & 6,6 & 10,3 & 113 & 109 \\
\hline Trihexafenidila & $0,2-5,0$ & $y=0,655 x+0,027$ & 0,9915 & 6,9 & 3,2 & 7,1 & 4,5 & 101 & 99 \\
\hline Venlafaxina & $0,2-5,0$ & $y=0,158 x+0,067$ & 0,9970 & 6,5 & 5,9 & 6,3 & 5,8 & 104 & 96 \\
\hline Zolpidem & $0,2-5,0$ & $y=0,297 x-0,023$ & 0,9910 & 7,9 & 5,5 & 6,8 & 5,9 & 104 & 106 \\
\hline
\end{tabular}

*CQB: Controle de qualidade alto. Concentração de $0,4 \mu \mathrm{g} \mathrm{mL}{ }^{-1}$, exceto para cafeína, carbamazepina e lidocaína onde a concentração é de $4 \mu \mathrm{g} \mathrm{mL}^{-1}$. **CQA: Controle de qualidade alto. Concentração de $4 \mu \mathrm{g} \mathrm{mL}^{-1}$, exceto para cafeína, carbamazepina e lidocaína onde a concentração é de $40 \mu \mathrm{g} \mathrm{mL}^{-1}$

Tabela 3. Resultados obtidos na análise de amostras de plasma de pacientes suspeitos de intoxicações

\begin{tabular}{|c|c|c|c|c|c|}
\hline Amostra & $t_{R R}$ & IR & Substância & Concentração $\left(\mu \mathrm{g} \mathrm{mL}^{-1}\right)$ & Concentração tóxica $\left(\mu \mathrm{g} \mathrm{mL} L^{-1}\right)^{8}$ \\
\hline 1 & 0,509 & 1582 & Femproporex & 0,21 & n.d. \\
\hline 2 & 0,936 & 2194 & Amitriptilina & 0,23 & $>0,5$ \\
\hline 3 & 0,982 & 2282 & Carbamazepina & 15,12 & $>10$ \\
\hline 4 & 0,982 & 2282 & Carbamazepina & 20,51 & $>10$ \\
\hline 5 & 0,982 & 2282 & Carbamazepina & 8,63 & $>10$ \\
\hline 6 & 0,982 & 2282 & Carbamazepina & 15,44 & $>10$ \\
\hline 7 & 0,982 & 2282 & Carbamazepina & 5,88 & $>10$ \\
\hline 8 & 0,982 & 2282 & Carbamazepina & 7,95 & $>10$ \\
\hline
\end{tabular}

n.d.: não disponível na literatura 
IR nas amostras clínicas apresentaram grande similaridade com os valores obtidos na validação do método. No entanto, o uso de IR nas condições instrumentais empregadas permite ampliar o número de substâncias potencialmente identificadas através da comparação com um amplo banco de dados já disponível, ${ }^{9}$ cuja comparabilidade com os resultados obtidos foi demonstrada neste estudo.

\section{CONCLUSÕES}

Foi desenvolvido e validado um método para identificação e quantificação de 40 fármacos básicos em plasma humano empregando extração líquido-líquido, sem etapa de evaporação de solvente, seguida de análise por cromatografia gasosa com detector de nitrogênio e fósforo. A retenção dos compostos foi caracterizada através de seus índices de retenção, os quais apresentaram excelente reprodutibilidade e, especialmente, comparabilidade com amplas bases de dados já disponíveis. O método mostrou-se apropriado para aplicação em toxicologia de emergência devido a sua sensibilidade, rapidez e especificidade.

\section{MATERIAL SUPLEMENTAR}

Está disponível em http://quimicanova.sbq.org.br, na forma de arquivo PDF e com acesso livre.

\section{AGRADECIMENTOS}

Este estudo foi apoiado pelo Conselho Nacional de Desenvolvimento Científico e Tecnológico (CNPq) e pela Fundação de Amparo à Pesquisa do Estado do Rio Grande do Sul (FAPERGS). Ao Instituto de Toxicologia da PUCRS pelo fornecimento de materiais empregados no estudo.

\section{REFERÊNCIAS}

1. Linden, R.; Feltraco, L. L.; Comerlato, L. C.; Kellermann, E.; Antunes, M. V.; Forensic Sci. Int. 2010, 202, 53.
2. Manca, D.; Ferron, U.; Weber, J.; Clin. Chem. 1989, 35, 601

3. Drummer, O. H.; Horomidis, S.; Koutis, S.; Syrjanen, M. L.; Tippet, P.; J. Anal. Toxicol. 1994, 18, 134.

4. Rasanen, I.; Ojanpera, I.; Vartiovaara, J.; Vuori, E.; Sunila, P.; J. High Resol. Chrom. 1996, 19, 313.

5. Torre, C. S.; Martínez, M. A.; Almarza, E.; Forensic Sci. Int. 2005, 155, 193.

6. Rasanen, I.; Kontinen, J.; Nokua, I.; Ojanperä, E.; J. Chromatogr., B: Anal. Technol. Biomed. Life Sci. 2003, 788, 243.

7. Gergov, M.; Nokua, J.; Rasanen, I.; Ojanpera, I.; Prob. Forensic Sci. 2000, 43. 70.

8. Turcant, A.; Premel-Cabic, A.; Cailleux, A.; Allain, P.; Clin. Chem. 1988, 34, 1492.

9. Zeeuw, R. A.; Franke, J. P.; Maurer, H. H.; Pfleger, K.; Gas chromatographic retention indices of toxicologically relevant substances on packed or capillary columns with dimethylsilicone stationary phases, $3^{\text {rd }}$ ed., VCH: Weinheim, 1992.

10. Schulz, M.; Schmoldt, A.; Pharmazie 2003, 58, 447.

11. Kirchherr, H.; Kuhn-Velten, W. N.; J. Chromatogr, B: Anal. Technol. Biomed. Life Sci. 2006, 843, 100.

12. Remane, D.; Meyer, M. R.; Peters, F. T.; Wissenbach, D. K.; Maurer, H. H.; Anal. Bioanal. Chem. 2010, 397, 2303.

13. Bassan, D. M.; Erdmann, F.; Krull, R.; Anal. Bioanal. Chem. 2011, 400, 43.

14. Remane, D.; Meyer, M. R.; Wissenbach, D. K.; Maurer, H. H.; Anal. Bioanal. Chem. 2011, 401, 1341

15. Wu, A. H. B.; McKay, C.; Broussard, L. A.; Hoffman, R. S.; Kwong, T. C.; Moyer, T. P.; Otten, E. M.; Welch, S. L.; Wax, P.; Clin. Chem. 2003, $49,357$.

16. Shah, V. P.; Midha, K. K.; Findlay, J. W. A.; Hill, H. M.; Hulse, J. D.; McGilveray, I. J.; McKay, G.; Miller, K. J.; Patnaik, R. N.; Powell, M. L.; Tonelli, A.; Viswanathan, C. T.; Yacobi, A.; Pharm. Res. 2000, 17, 1551.

17. Linden, R.; Kellermann, E.; Sartori, S.; Souto, A. A.; Quim.Nova 2007, 30,468 . 